\title{
UTILIZAÇÃO DO SIMULADOR DE PROCESSOS IDEAS EM VALIDAÇÃO DE LÓGICAS DE CONTROLE E TREINAMENTO OPERACIONAL*
}

\section{Resumo}

Paulo Henrique Zunzarrem Pinto ${ }^{\dagger}$ Henrique Paier Milanez $z^{2}$

Taiguara Melo Tupinambás ${ }^{3}$

Dois assuntos que vêm sendo cada vez mais discutido na indústria são revisão crítica de projetos e a capacitação da equipe de operação para otimização do processo. Além dos benefícios financeiros diretos que se obtêm na redução do tempo de partida, as melhorias, quando identificadas em fases anteriores do projeto, produzem resultados positivos durante outras fases do ciclo de vida da planta. Neste sentido, o sistema de controle e a capacitação dos operadores desempenham um papel fundamental. $O$ presente trabalho apresenta uma estratégia efetiva de se validar as lógicas de controle de uma planta por meio do simulador dinâmico de processos de alta fidelidade IDEAS, e a utilização desta ferramenta no treinamento operacional via OTS (Operator Training Simulator). Neste trabalho, é realizada uma breve revisão sobre a metodologia utilizada para a construção da arquitetura de automação que permitiu a validação das lógicas de controle e o desenvolvimento do sistema de treinamento. Especificamente, são apresentados resultados obtidos com a simulação em projetos de beneficiamento mineral, por meio da quantificação dos ganhos em operação, manutenção e segurança. Como exemplo de resultados, mais de 2000 pontos de melhoria foram encontrados em projetos executados, e mais de 14 cenários de operação foram criados para treinamento. Os ganhos observados corroboram a robustez da ferramenta utilizada e o potencial da metodologia empregada.

Palavras-chave: IDEAS; Simulação dinâmica; Treinamento operacional; Validação de lógicas de controle.

\section{THE ROLE OF IDEAS PROCESS SIMULATOR ON CONTROL LOGIC VALIDATION AND OPERATIONAL TRAINING SYSTEMS}

\section{Abstract}

Two subjects that have been drawing increased attention in the mining industry are project design review and operational team training for optimization. Besides direct financial benefits resulting from reduced startup time, these improvements - when they are identified during project phases - will provide added benefits during other phases of the plant lifecycle. As such, the control system and operator training play an essential and fundamental role in project development. This paper presents an effective way to validate a plant's control logic and train operators by using IDEAS, a high-fidelity dynamic simulator. The presentation will include a brief review of the methodology used for the construction of the automation architecture, which in an important factor in enabling control system validation and the development of the training system. Specific results obtained by the simulation in mineral processing projects are discussed, including the quantification of gains in operation, maintenance, and safety. As an example of results, more than 2,000 improvement points were found in five executed projects and more than 14 operational scenarios were created for training. The results achieved in those projects corroborate the tool's robustness and the potential of the methodology. Keywords: IDEAS; Dynamic simulation; Operational training; Control logic validation.

Analista de Automação, ANDRITZ Brasil, Belo Horizonte, Minas Gerais, Brasil.

Engenheiro de Processos, B.Sc., ANDRITZ Brasil, Belo Horizonte, Minas Gerais, Brasil.

Engenheiro Eletricista, B.Sc., ANDRITZ Brasil, Belo Horizonte, Minas Gerais, Brasil. 


\section{INTRODUÇÃO}

\subsection{Simulação de Processos}

O termo simulação pode ser entendido como o processo de modelagem e análise de um sistema ou processo real, visando descrever seu comportamento. Ou seja, por meio da simulação, é possível elaborar hipóteses sobre um sistema, propor e conduzir testes, e usar os resultados para avaliar diferentes estratégias de operação e os efeitos que as mesmas terão sobre o processo [1].

O simulador de processos IDEAS possui uma interface gráfica baseada em objetos, na qual o usuário monta seu modelo escolhendo objetos de diferentes bibliotecas e os conectando por meio de linhas de conexão. O software IDEAS pode realizar um balanço de massa e energia, acompanhar fluxo e concentração de componentes, compostos e elementos, identificar variações na distribuição granulométrica, assim como calcular densidade, entalpia. Operações unitárias simuladas em IDEAS usam princípios elementares, configurando a base para 0 dimensionamento de equipamentos do processamento mineral. Além dos princípios básicos, IDEAS também possui equações semi-empíricas e correlações específicas para determinadas operações unitárias que não podem ser previstas por equações matemáticas embasadas em princípios físicos fundamentais [2].

\subsection{Aplicação da Simulação de Processo}

Um simulador dinâmico como IDEAS pode ser utilizado em todas as etapas do ciclo de vida de um projeto de uma planta de processamento de minério, isto é, desenvolvimento de processos, validação de engenharia, dimensionamento dos equipamentos, ajuste de malhas de controle, teste das lógicas de controle e treinamento operacional [2].

Experiência em projetos tem determinado que a validação das lógicas de controle utilizando um simulador dinâmico de processos pode eliminar até em $80 \%$ dos erros de programação do sistema de controle antes da planta ser comissionada. Utilizar modelos dinâmicos têm se mostrado salvar tempo e dinheiro em inúmeras unidades fabris de diversas indústrias, tais como mineração e papel e celulose. Como um exemplo, em um caso, o startup foi executado em 16 dias ao invés de 90 dias e estava acima da capacidade de produção da planta em um mês. Em outro caso, o tempo no comissionamento foi adiantado em três semanas [3].

Um simulador dinâmico de processos como o IDEAS ajuda no processo de aprendizagem, envolvendo os operadores e dando feedbacks imediatos sem riscos a produção. Também, um sistema de treinamentos com devida manutenção e atualização pode ser utilizado para treinar novos operadores, ajudando-os rapidamente a se tornarem qualificados para a operação do processo [3].

O presente trabalho tem por objetivo demonstrar como um simulador dinâmico como - IDEAS vem sendo utilizado para validação de lógicas de controle e treinamento operacional. Ainda, serão apresentados estudos de casos em que a utilização da simulação de processos para estes fins proporcionou diversos ganhos em operação, manutenção, segurança, tempo de startup e ramp-up, além dos cenários treinados. 


\section{MATERIAIS E MÉTODOS}

\subsection{Ciclo de Vida de um Projeto de Simulação}

Um projeto de simulação dinâmica pode ser dividido em duas etapas distintas e em subetapas dependendo do objetivo final da simulação [3]. Na Figura 1 são apresentadas estas etapas típicas conforme o desenvolvimento do projeto.

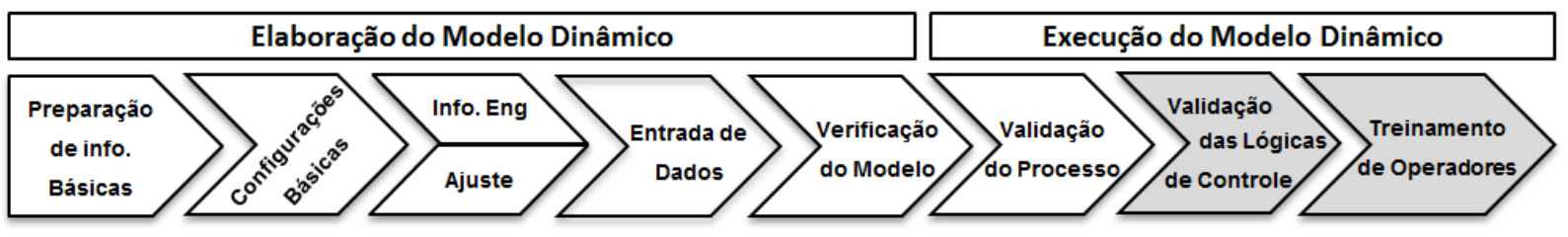

Figura 1. Típico ciclo de vida de um projeto de simulação dinâmica

A primeira etapa de execução do modelo dinâmico (aqui nomeada como validação do processo) nada mais é do que a representação de todos os dados de engenharia detalhada de uma forma integrada, funcionando como um fluxograma de tubulação e instrumentação (P\&ID) virtual [3]. Após esta fase, inicia-se a fase de validação das lógicas de controle. Esta fase, aqui designada comissionamento virtual, é feita normalmente com uma equipe de profissionais treinados para funções específicas: o engenheiro de automação responsável pela configuração e implementação do sistema de controle da planta, o engenheiro de simulação responsável pelo adequado funcionamento de um modelo dinâmico fidedigno ao projeto e a equipe operacional de painel que será responsável pela partida da planta. As etapas típicas do comissionamento virtual (validação das lógicas de controle) estão especificadas na Figura 2.

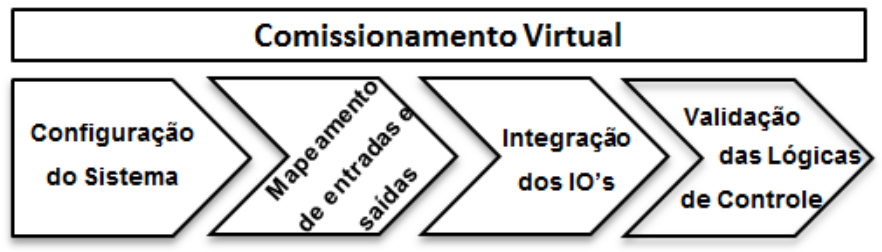

Figura 2. Típicas Etapas desenvolvidas durante a validação das lógicas de controle

A terceira e última fase da execução do modelo dinâmico é o treinamento operacional. Nesta fase, além dos procedimentos de partida e parada de equipamentos e acompanhamento de produção, são criados cenários de processo para desenvolver a ação da operação. Esses cenários podem simular problemas elétricos (como um "apagão"), de processo (como mudança da dureza e qualidade do minério), de instrumentação (mau funcionamento de elementos de medição) ou ainda mecânicos (como válvula travada ou entupimento da tubulação).

O objetivo global de todas estas etapas é aumentar a disponibilidade operacional da planta, por meio da melhoria do projeto de engenharia, de facilitação na implementação do sistema de controle e operadores altamente qualificados [4]. No presente trabalho são apresentados os métodos e resultados das fases de validação das lógicas de controle e do treinamento operacional. 


\subsection{Criação da Plataforma Virtual}

A criação da plataforma virtual pode ser dividida em quatro etapas distintas: a configuração do sistema, o mapeamento de entradas e saídas e construção dos típicos de acionamento de motores, a integração dos pontos de comunicação com o processo (P\&ID virtual) e validação das lógicas de controle.

Durante a configuração do sistema, garante-se a comunicação entre os softwares de simulação, de emulação do sistema de controle e sistema de supervisão utilizando como meio de comunicação o padrão OLE for Process Control (OPC). Dessa forma, toda instalação, licenciamento e configuração do sistema operacional, dos softwares e dos serviços são realizados nessa etapa. Para isso, é necessária a montagem da arquitetura do sistema de automação conforme a Figura 3.

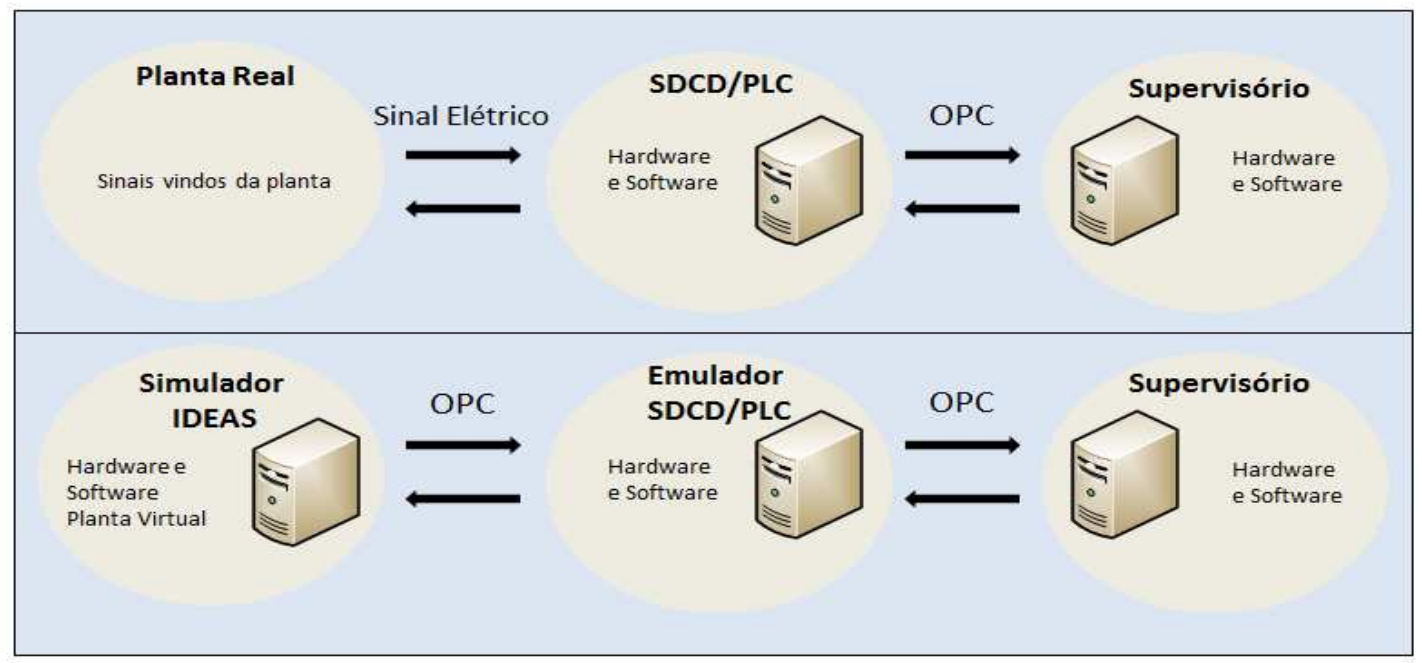

Figura 3. Arquitetura de automação da plataforma virtual

$\mathrm{Na}$ segunda etapa de criação da plataforma virtual, os pontos de conexão com o sistema de controle são criados no simulador IDEAS (semelhante ao comissionamento realizado em campo, que visa garantir a comunicação entre os pontos digitais e analógicos com o sistema de supervisão). Para tanto, é necessária a criação de todas as entradas/saídas digitais e analógicas no IDEAS. Em cada bloco de comunicação (entrada ou saída) do IDEAS é configurado o endereço de comunicação OPC de acordo com a configuração do sistema de controle. Os blocos responsáveis pelo envio e recebimento de sinais via OPC estão representados na Figura 4.

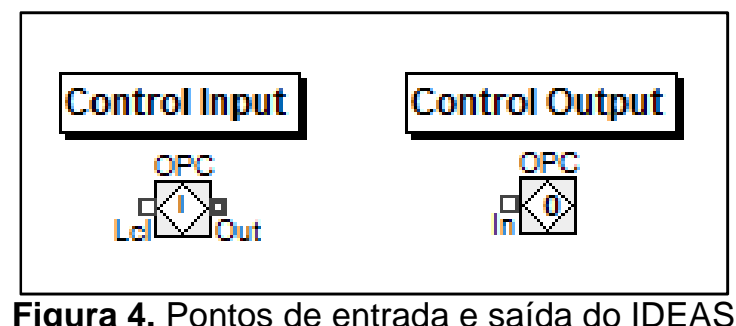

Figura 4. Pontos de entrada e saída do IDEAS

Os típicos de acionamento (representação logica do funcionamento dos sistemas de proteção de campo nos sistemas de controle) são responsáveis por envio e recebimento das palavras (words) de comunicação. Estas palavras são divididas em bits para que as informações pré-configuradas nos relés sejam extraídas. Dessa forma, faz-se necessário o desenvolvimento desta comunicação no IDEAS. Para 
tanto, são utilizados blocos já pré-programados para manipulação de sinais. São mostrados na Figura 5 alguns exemplos dos blocos utilizados no IDEAS para envio e recebimento de sinais (blocos verdes retangulares).

O processo de desenvolvimento dos típicos exige certo conhecimento do sistema de controle da planta em questão e do simulador de processos. Estando claro como funcionam todos os típicos de acionamento da planta e sabendo manipular os blocos do IDEAS, pode-se construir uma comunicação robusta entre os softwares, sendo que após a configuração é possível simular qualquer sinal de falha do campo com a ajuda do simulador. Adicionalmente, relés que possuem lógicas para falhas em partidas consecutivas (após três partidas em menos de uma hora o motor entra em falha), que enviam o tempo de operação do motor e disponibilizam a temperatura em diferentes pontos do motor, por exemplo, podem ser facilmente configurados e, então, simulados. Um exemplo de representação é mostrado na Figura 5.

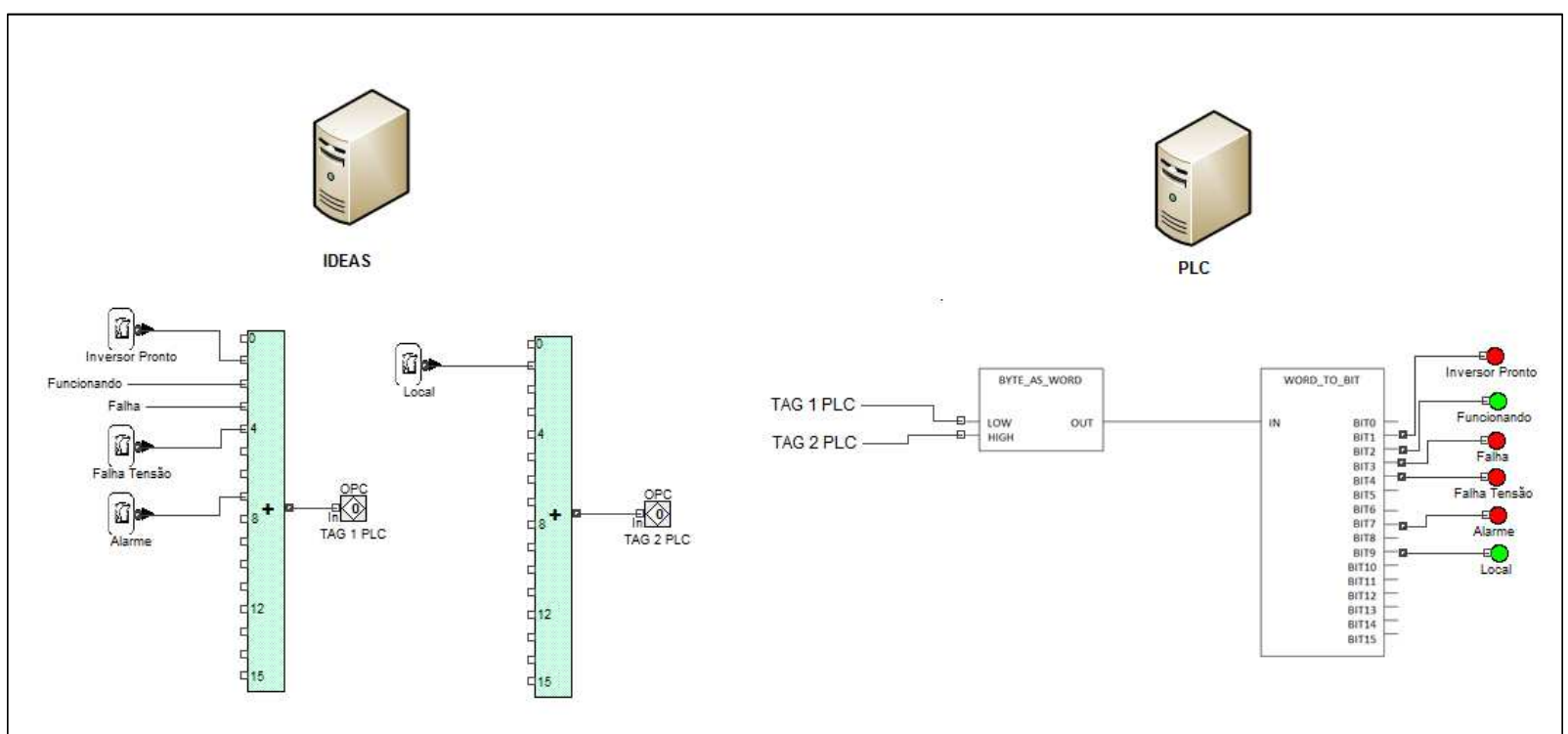

Figura 5. Envio e recebimento de sinais em típicos de acionamento de motos no IDEAS

Após o mapeamento de todas as entradas e saídas e construção de todos os típicos de acionamento de motores, inicia-se a etapa de integração dos pontos de comunicação com o processo. Nessa fase, todos os típicos de acionamento criados são conectados aos blocos correspondentes, de modo que o quando acionado o motor de uma bomba pelo supervisório, o mesmo irá enviar um sinal de "liga" para o IDEAS, fazendo com que o objeto (Pump Centrifugal w/Motor) comece a realizar os cálculos da bomba em funcionamento. Após o mapeamento, realiza-se um startup e shutdown da planta para testar se a plataforma criada responde corretamente.

Por fim, a plataforma virtual está criada. Estando configurada, testada e pronta para a validação das lógicas de controle e treinamento operacional.

\subsection{Validação das Lógicas de Controle}

Com a plataforma virtual criada, associada ao modelo dinâmico, podem-se iniciar os testes das lógicas de controle. Na Figura 6, é mostrado um exemplo do modelo no IDEAS pronto para a validação. Os pontos vermelhos da Figura 6 são pontos de comunicação entre IDEAS e o Computador Lógico e Programável (PLC), sendo que podem ser entradas ou saídas, digitais ou analógicas. 


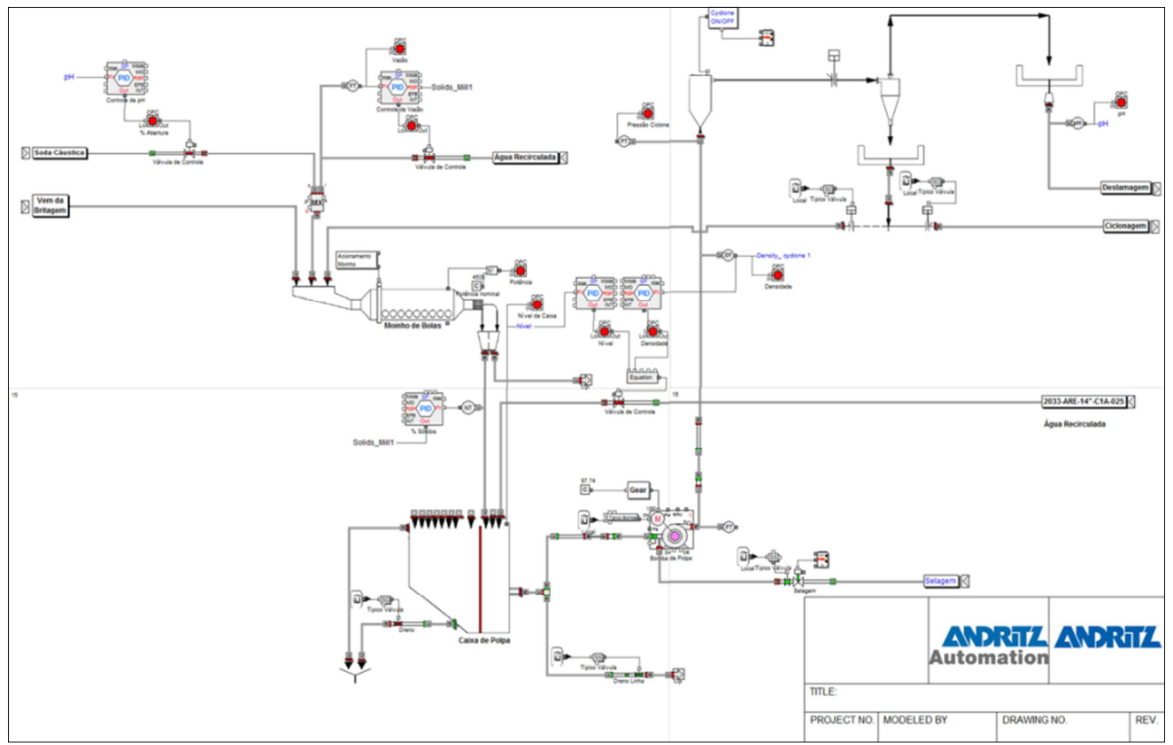

Figura 6. Envio e recebimento de sinais em típicos de acionamento de motos no IDEAS

Com a metodologia de teste por malha de controle, sendo testado uma por uma, o simulador de processos é utilizado não somente para os tradicionais testes de plataforma, mas também para uma análise muito mais profunda e ampla do sistema de controle. Validar as lógicas de controle por meio de um modelo dinâmico detalhado permite detectar erros que envolvem processo (como resposta da pressão, temperatura, respostas de bombas e válvulas), além dos tradicionais erros que são encontrados nos testes de plataforma [3].

Os testes são executados em uma sequência lógica, muitas vezes definida pelo técnico de operação da planta. Em projetos anteriores os testes eram feitos seguindo a sequência da Tabela 1 . Todos os pontos levantados são compilados em uma planilha em forma de punchlist categorizando e quantificando os resultados obtidos.

Tabela 1. Etapas de validação das lógicas de controle

\begin{tabular}{ll}
\hline \multicolumn{1}{c}{ Etapa de Validação de Lógicas } \\
\hline 1) Teste de IO’s e Típicos de Acionamento \\
2) Avaliação dos Intertravamentos de Processo \\
3) Avaliação das lógicas de Partida e Parada \\
4) Teste das Malhas no Processo \\
5) Sintonia de Controladores \\
6) Verificação de Estratégias de Controle Avançado \\
\hline
\end{tabular}

\subsection{Treinamento Operacional}

Uma vez que o sistema de controle é validado e está comunicando com o modelo da planta, é possível utilizá-lo como uma ferramenta para treinamento de operadores, solução conhecida como Operator Training Simulator (OTS). Durante o treinamento, o operador usa o próprio sistema de controle que irá ser utilizado na partida e operação da planta. Ainda, utiliza a mesma tela supervisório para interagir com o processo. Para o treinamento, são criados diversos cenários de operação em cooperação entre cliente e fornecedor, que irão refletir situações reais que poderão ser encontradas pelos operadores na planta real. 
Na Tabela 2 são mostradas as diferentes formas de aprendizado possibilitadas pelo OTS.

Tabela 2. Exemplos de treinamentos utilizando IDEAS

\begin{tabular}{ll}
\hline \multicolumn{1}{c}{ Treinamento Operacional } \\
\hline 1) Aprender o procedimento de partida e parada \\
2) Aprender a diagnosticar e corrigir variações de processo e falhas em \\
equipamentos \\
3) Aprender procedimentos de emergência \\
4) Aprender a dinâmica real do processo e desenvolver uma noção de tempo de \\
resposta do processo \\
5) Ganhar conhecimento de processo e interação entre equipamentos \\
\hline
\end{tabular}

\subsection{Avaliação de Desempenho}

Parte fundamental do projeto é a avaliação de desempenho dos operadores, que se dá antes do treinamento se iniciar e após sua conclusão, para fins comparativos. Esta avaliação tem como base testes teóricos e o próprio desempenho na operação do sistema, levando em consideração os cenários desenvolvidos e a operação por períodos específicos. A cada uma destas atividades são atribuídos pesos e, no final, as notas variam de 1 a 100. A avaliação pode ser dividida em competências ou áreas de processo, para um diagnóstico mais preciso de competências.

É importante ressaltar, no entanto, que a identificação e a correção dos problemas de desempenho serão obtidas durante toda etapa do treinamento.

\section{RESULTADOS E DISCUSSÃO}

Os dados aqui apresentados são resultados obtidos de cinco diferentes projetos na área de beneficiamento mineral. Estes possuem como escopo todo o ciclo de vida do projeto de simulação sendo aqui apresentados somente os resultados das fases de validação das lógicas de controle e treinamento operacional.

\subsection{Validação das Lógicas de Controle}

Durante a validação das lógicas de controle, foram encontradas melhorias em três áreas: operação, manutenção e segurança. Estes foram categorizados de acordo com o tipo de ganho e são demonstrados na Tabela 3.

Tabela 3. Categorização dos resultados obtidos em validação das lógicas de controle

\begin{tabular}{|c|c|c|}
\hline OPERAÇÃO & MANUTENÇÃO & SEGURANÇA \\
\hline $\begin{array}{ll}\text { - } & \text { Redução do tempo de startup } \\
\text { - } & \text { Otimização da partida } \\
\text { - } & \text { Aumeção do tempo de parada } \\
& \text { informaçãol de } \\
\text { - } & \text { Previne transbordo } \\
\text { - } & \text { Facilita a operação }\end{array}$ & $\begin{array}{l}\text { - } \quad \text { Evita dano ao equipamento } \\
\text { - Relhora nível de informação } \\
\text { Reduz downtime }\end{array}$ & $\begin{array}{ll}\text { - } & \text { Evita exposição ao } \\
\text { risco } \\
\text { - } \\
\text { Maior segurança } \\
\text { pessoal }\end{array}$ \\
\hline
\end{tabular}

Observa-se que os benefícios vão além do objetivo principal do investimento, redução do tempo de startup. Além disso, durante a etapa conceitual do projeto, imaginou-se que os ganhos seriam concentrados apenas na operação. Entretanto, com o alto nível de fidelidade do modelo, foi possível estender os resultados para 
outras áreas, como manutenção e segurança. Como um exemplo, em um dos projetos, foi observado que da forma com o qual um alarme de nível alto foi parametrizado, não seria possível para a operação fazer nenhuma manobra sem que o silo transbordasse. Sendo assim, a lógica foi alterada garantindo a segurança na área. Em outro momento, foi detectada a necessidade de modificação das logicas de intertravamento do alimentador que envia material ao britador. A modificação fezse necessária para impedir que o equipamento sofresse dano, uma vez que sem esta, o britador iria parar cheio.

Foram encontrados também problemas de menor impacto ao processo que também podem ser citados, como, ajuste das malhas de controle, reparo dos endereços de comunicação (entre sistemas de supervisão e controle), melhoria de animação nas telas de supervisão, ajustes de lógicas de parada e partida automática, dentre outros.

$\mathrm{Na}$ Tabela 4, é apresentado um resumo dos resultados extraídos dos punchlists de cada projeto e o percentual de melhorias por tipo de benefício.

Tabela 4. Distribuição e totalização dos pontos de melhorias encontrados

\begin{tabular}{ccccc}
\hline Projeto & № de Melhorias & Operação & Manutenção & Segurança \\
\hline 1 & 96 & $61 \%$ & $17 \%$ & $22 \%$ \\
2 & 186 & $52 \%$ & $26 \%$ & $22 \%$ \\
3 & 427 & $56 \%$ & $27 \%$ & $17 \%$ \\
4 & 578 & $53 \%$ & $33 \%$ & $13 \%$ \\
5 & 1068 & $73 \%$ & $17 \%$ & $10 \%$ \\
Total Melhorias & 2355 & $59 \%$ & $24 \%$ & $17 \%$ \\
\hline
\end{tabular}

Como resultado do sistema de treinamento, foi possível obter uma visão dos pontos que demandavam uma maior atenção no processo, além do fornecimento de diretrizes para a solução de problemas e melhorias. Os números da Tabela 4 demonstram que a maior parte dos pontos de problema/melhoria encontrados estão relacionados diretamente a equipe de operação. Sendo assim, podemos afirmar que os resultados impactam diretamente na produção, segurança e manutenção da planta.

\subsection{Treinamento Operacional}

É possível observar benefícios já na etapa de criação dos cenários de operação, uma vez que, para isso, é necessário que os operadores envolvidos no projeto discutam os cenários e criem procedimentos operacionais para lidar com cada um deles. Estes documentos são desenvolvidos com o intuito de informar e documentar as ações corretas a serem tomadas durante um dos eventos simulados. Desta forma, é nítido o ganho em conhecimento por parte destes operadores, além da contribuição com a documentação do projeto, uma vez que o material elaborado contribui como base para o treinamento no simulador e, posteriormente, como material de consulta.

Nas Tabelas 5 e 6, exemplos de cenários podem ser observados para as áreas de Cominuição e Beneficiamento, respectivamente. 
Tabela 5. Cenários da Cominuição

\section{Cenários - Cominuição}

- Interrupção do fornecimento de energia elétrica

- Mudança na distribuição granulométrica do ROM

- Balança de alimentação Descalibrada

- Executar sequência para manutenção preventiva

- Aumento da carga circulante devido a mudança de granulometria

Tabela 6. Cenários do Beneficiamento

\begin{tabular}{l} 
Cenário - Beneficiamento \\
\hline - Produto da Moagem fora da especificação \\
- Distúrbio nos instrumentos de Densidade \\
- Distúrbio nos instrumentos de Pressão de Ciclones \\
- Torque eleveia na Vazão de ar para células de Flotação \\
- Entupimento na tubulação \\
- Dalta de Água nos reservatórios \\
\hline
\end{tabular}

Já a Tabela 7 apresenta as ações necessárias para um cenário de blackout.

Tabela 7. Procedimentos operacionais

\begin{tabular}{|c|c|c|}
\hline Cenário & Ação Esperada & Como Executar \\
\hline $\begin{array}{c}\text { Interromper } \\
\text { Fornecimento de } \\
\text { Energia Elétrica }\end{array}$ & $\begin{array}{ll}\text { - } & \text { Verificar equipamentos atingidos } \\
& \text { Verificar se equipamentos } \\
\text { - } & \text { Rearmarm com material } \\
\text { - } & \text { Rodar em local os equipamentos } \\
& \text { parados com material } \\
& \text { Partida }\end{array}$ & $\begin{array}{ll} & \text { Tela do Supervisório } \\
\text { - } & \text { Operador de Campo via rádio } \\
\text { - } & \text { Passar equipamentóntos para Local } \\
\text { Tela do Supervisório }\end{array}$ \\
\hline
\end{tabular}

Como exemplo de resultado das avaliações de desempenho, são apresentadas na Figura 7, as notas do diagnóstico inicial e da avaliação final, após a conclusão dos treinamentos, onde é possível observar a evolução do aprendizado daquela turma específica.

Ficou claro ao término do treinamento, que os operadores tomavam decisões com muito mais segurança e tinham um conhecimento muito maior da planta, permitindo uma operação mais segura, mais produtiva, mais eficiente e com produtos de melhor qualidade. 


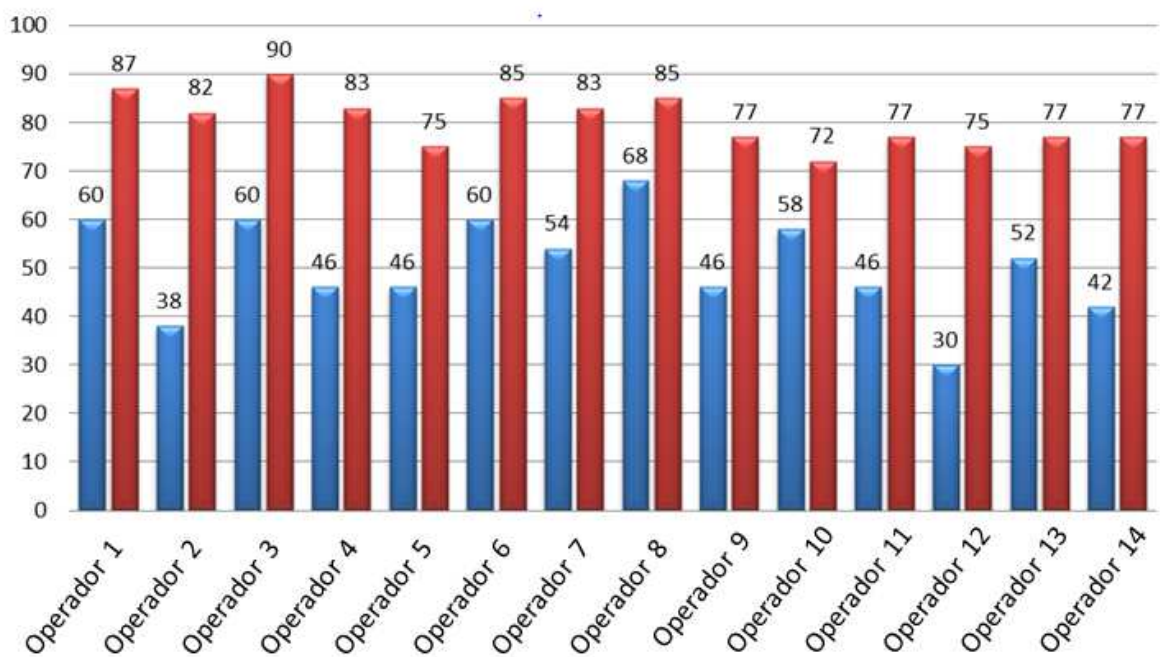

Figura 7. Gráfico de desempenho. Azul é o diagnóstico inicial e vermelho o final

\section{CONCLUSÃO}

Como se pode observar, a simulação dinâmica de processos pode ser utilizada em diversas fases do projeto, em especial, nas fases de validação das lógicas de controle e treinamento operacional.

No presente trabalho, foram apresentados resultados de cinco projetos realizados pela ANDRITZ AUTOMATION, sendo que foram encontrados mais de 2000 pontos de melhorias, representando uma indiscutível redução no startup. Ainda, foi possível a criação de cenários de processo para treinamento da equipe operacional, com foco em operação, manutenção e segurança. Com isso, as lógicas de controle foram testadas e corrigidas em uma plataforma virtual muito próxima ao processo real e a operação treinada e ambientada ao sistema de operação da planta.

Com a devida manutenção, o sistema de treinamento pode ainda ser uma excelente forma de consulta do processo, sendo possível treinar novos operadores, estudar novas formar de controle e avaliar investimentos para otimização do processo, tornando-se uma ferramenta interessante, também, para plantas em operação.

\section{REFERÊNCIAS}

1 Centeno, M., "An Introduction to Simulation Modelling," 1996.

2 Parthasarathi, P., Szaruga, V., and Szatkowski, M., Benefits of Dynamic Process Simulation for Mineral Industry, 2009. Disponível em http://www.andritz.com/aaautomation-benefits-dynamicprocesssimulation-mineral.pdf.

3 Milanez, H., Villela, R., and Lorentz, S., Benefits of dynamic simulation for mineral industry: Validating engineering diagrams with IDEAS dynamic simulator, 18th Seminário de Automação e TI Industrial, September 23-26, 2014, Sao Paulo, Brazil.

4 Schug, B.W., Nees, M.R., and Gamarano, T.V., Process Simulation for Improved Plant Design through P\&ID Validation, presented at the SME, Seattle, Washington, USA, February 22, 2012. Disponível em http://www.andritz.com/ aa-automation-simulationplantdesign-p_id-validation.pdf. 